\title{
Low Energy Leptogenesis in Left-Right Symmetric Models
}

\author{
Avijit Ganguly, Jitendra C. Parikh and Utpal Sarkar † \\ Theory Group, \\ Physical Research Laboratory, \\ Ahmedabad - 380009, India.
}

August 1994

\begin{abstract}
We propose a new mechanism for baryogenesis. We study the effective potential of left-right extension of the standard model and show that there can be a first order phase transition at the left-right symmetry breaking and hence $(B-L)$ symmetry breaking scale, which is around $\mathrm{TeV}$ in our scenario. As a result, although $(B-L)$ violating interactions are in equilibrium at this scale, enough $(B-L)$ asymmetry may be generated. This $(B-L)$ asymmetry is then converted to baryon asymmetry during the anomalous electroweak process. If right handed gauge bosons are seen in the $\mathrm{TeV}$ scale, then we argue that this will be the only consistent mechanism to generate baryon asymmetry of the universe.
\end{abstract}

\footnotetext{
${ }^{1}$ Address during March 1994 - February 1995 : Institut für Physik, Universität Dortmund, D-44221 Dortmund, Germany.
} 
In grand unified theories baryogenesis is generated through $(\mathrm{B}+\mathrm{L})$ asymmetry of the universe [1, 2], since $(B-L)$ is either a global symmetry (as in the case of $S U(5)$ GUT) or a local symmetry (as in the $S O(10)$ or $E(6)$ GUT) [2]. The sphaleron field at high temperature will then erase this primordial $(B+L)$ asymmetry during the electroweak phase transition.

It was then proposed that since $C$ and $C P$ are not conserved in the standard model and there is baryon number violation due to the anomaly [3], it is possible to generate enough baryon number [4, 5] if the electroweak phase transition is of weakly first order. But for this generated baryon asymmetry to survive after the phase transition one requires that the higgs mass has to be quite low (around $50 \mathrm{GeV}$ ) [6], while the present experimental lower bound is around $70 \mathrm{GeV}$. This makes this interesting scenario impracticable.

Then the only viable possibility open to us is to generate $(B-L)$ asymmetry at higher energies, which will then get converted to baryon asymmetry during the electroweak phase transition in the presence of the sphaleron field, when the $(B+L)$ violating anomalous processes are in equilibrium in the universe [7, 8]. Models were proposed where the lepton number violating out-of-equilibrium interactions of the right handed neutrinos and other scalar particles generate the required $(B-L)$ asymmetry [7]. $C$ and $C P$ violation of correct magnitude is not a problem in such models [9].

Although in the original model [7] the standard model was extended to have a global $(B-L)$ symmetry and sterile neutrinos were added, leptogenesis comes about more naturally in left-right symmetric models [8], where $(B-L)$ is a local symmetry. Furthermore, left-right symmetric models 10 are the most natural extension of the standard model and are phenomenologically very attractive particularly if they are broken at around $\mathrm{TeV}$ scale. In that case in the next generation accelerators we shall see signatures of the existence of the extra gauge particles which mediate interactions among the right handed particles. On the other hand, as we shall see later, the out- 
of-equilibrium condition imposes a lower bound on the left-right symmetry breaking scale [11,

$$
M_{R} \geq 10^{7} \mathrm{GeV} .
$$

Will that mean that if $Z_{R}$ is seen in the near future, then that will rule out the possibility of leptogenesis completely and we have to look for new solutions for baryogenesis?

In this paper, we propose a new mechanism for baryogenesis through lepton number violation in which the left-right symmetry and hence $(B-L)$ symmetry is broken at around $\mathrm{TeV}$ scale [12]. We shall show that this phase transition can be weakly first order. As a result, during this phase transition bubbles of true vacuum will be formed inside which the $C P$ non-conserving lepton number violating and hence $(B-L)$ violating interactions will be in equilibrium, but outside the bubbles $(B-L)$ violating interactions can transmit from one bubble to the other along the bubble walls only when the bubbles collide. This will not allow the particles and antiparticles to attain equilibrium distribution even for $M_{R} \sim \mathrm{TeV}$ and hence enough $(B-L)$ asymmetry may be generated through the lepton number violating interactions. Finally during the electroweak phase transition, this $(B-L)$ asymmetry will be converted to baryon asymmetry of the universe via sphaleron effects.

For our purpose we consider the symmetry breaking chain, $S U(3)_{c} \times$ $S U(2)_{L} \times S U(2)_{R} \times U(1)_{(B-L)}\left[\equiv G_{L R}\right] \stackrel{M_{R}}{\longrightarrow} S U(3)_{c} \times S U(2)_{L} \times U(1)_{Y}\left[\equiv G_{\text {std }}\right]$ $\stackrel{M_{W}}{\longrightarrow} S U(3)_{c} \times U(1)_{e m}$. The symmetry breaking $G_{L R} \rightarrow G_{s t d}$ takes place when the right handed triplet higgs field $\Delta_{R} \equiv(1,1,3,-2)$ acquires a vacuum expectation value $(v e v)$. In this model $(B-L)$ is a local symmetry. The breaking of the group $G_{L R}$ also implies spontaneous breaking of $(B-L)$. Left-right parity implies the existence of another higgs field $\Delta_{L}$ which transforms as $(1,3,1,-2)$ under $G_{L R}$. A higgs doublet field $\phi(1,2,2,0)$ breaks the electroweak symmetry and gives masses to the fermions. With this choice of higgs scalars and usual fermions of the standard electroweak model and the right handed 
neutrino (which is always present in left-right symmetric model), the Yukawa couplings are given by

$$
\mathcal{L}_{Y u k}=f_{i j} \overline{\psi_{i L}} \psi_{j R} \phi+f_{L i j} \overline{\psi_{i L}{ }^{c}} \psi_{j L} \Delta_{L}^{\dagger}+f_{R i j} \overline{\psi_{i R}{ }^{c}} \psi_{j R} \Delta_{R}^{\dagger}
$$

The scalar interactions can be written as,

$$
\begin{aligned}
V= & -\sum_{i, j} m_{i j}^{2} \operatorname{tr}\left(\phi_{i}^{\dagger} \phi_{j}\right)+\sum_{i, j, k, l} \lambda_{i j k l}\left[\operatorname{tr}\left(\phi_{i}^{\dagger} \phi_{j}\right) \operatorname{tr}\left(\phi_{k}^{\dagger} \phi_{l}\right)+\operatorname{tr}\left(\phi_{i}^{\dagger} \phi_{j} \phi_{k}^{\dagger} \phi_{l}\right)\right] \\
& -\mu^{2}\left(\Delta_{L}^{\dagger} \Delta_{L}+\Delta_{R}^{\dagger} \Delta_{R}\right)+\rho_{1}\left[\operatorname{tr}\left(\Delta_{L}^{\dagger} \Delta_{L}\right)^{2}+\operatorname{tr}\left(\Delta_{R}^{\dagger} \Delta_{R}\right)^{2}\right] \\
& +\rho_{2}\left[\operatorname{tr}\left(\Delta_{L}^{\dagger} \Delta_{L} \Delta_{L}^{\dagger} \Delta_{L}\right)+\operatorname{tr}\left(\Delta_{R}^{\dagger} \Delta_{R} \Delta_{R}^{\dagger} \Delta_{R}\right)\right]+\rho_{3} \operatorname{tr}\left(\Delta_{L}^{\dagger} \Delta_{L} \Delta_{R}^{\dagger} \Delta_{R}\right) \\
& +\sum_{i, j} \alpha_{i j}\left(\Delta_{L}^{\dagger} \Delta_{L}+\Delta_{R}^{\dagger} \Delta_{R}\right) \operatorname{tr}\left(\phi_{i}^{\dagger} \phi_{j}\right)+\sum_{i, j} \beta_{i j}\left[\operatorname{tr}\left(\Delta_{L}^{\dagger} \Delta_{L} \phi_{i} \phi_{j}^{\dagger}\right)\right. \\
& \left.+\operatorname{tr}\left(\Delta_{R}^{\dagger} \Delta_{R} \phi_{i}^{\dagger} \phi_{j}\right)\right]+\sum_{i, j} \gamma_{i j}\left[\operatorname{tr}\left(\Delta_{L}^{\dagger} \phi_{i} \Delta_{R} \phi_{j}^{\dagger}\right)+\operatorname{tr}\left(\Delta_{R}^{\dagger} \phi_{i} \Delta_{L} \phi_{j}^{\dagger}\right)\right]
\end{aligned}
$$

where we defined, $\phi_{1} \equiv \phi ; \quad \phi_{2} \equiv \tau_{2} \phi_{1}^{*} \tau_{2}$. The vacuum expectation values (vev) of the fields have the following form:

$$
\begin{aligned}
& <\phi>=\left(\begin{array}{cc}
k & 0 \\
0 & k^{\prime}
\end{array}\right) ; \quad\left\langle\Delta_{L}>=\left(\begin{array}{cc}
0 & 0 \\
v_{L} & 0
\end{array}\right) ;\right. \\
& <\tilde{\phi}>=\left(\begin{array}{cc}
k^{\prime} & 0 \\
0 & k
\end{array}\right) ; \quad<\Delta_{R}>=\left(\begin{array}{cc}
0 & 0 \\
v_{R} & 0
\end{array}\right) ;
\end{aligned}
$$

Following equation (1), $\Delta_{L, R}$ can decay into two neutrinos and $\Delta_{L, R}^{\dagger}$ into two antineutrinos. When the fields $\Delta_{L, R}$ acquire vev and the $(B-L)$ is spontaneously broken, there are new $(B-L)$ number violating interactions of the fields $\Delta_{L, R}$,

$$
\Delta_{L, R} \rightarrow \phi+\phi \quad \text { or } \quad \phi^{\dagger}+\phi^{\dagger}
$$


There are also other lepton number violating diagrams arising from the right handed neutrino decays, when the vev of $\Delta_{R}$ gives Majorana mass to $\nu_{R}$. The lepton number violating decays of $\nu_{R}$ are given by,

$$
\nu_{i R} \rightarrow l_{j L}+\bar{\phi} \quad \text { or } \quad l_{j L}^{c}+\phi
$$

The excess lepton number however can only be generated, if in addition to the lepton number violation there is $C P$ violation. In principle, there can be, a rephasing invariant, complex phase in the Yukawa couplings, which can give $C P$ violation. The lepton number generation can then occur only through the interference of the one loop diagrams with the tree level interactions mentioned above. The relevant tree level and one loop diagrams are shown in fig. 1. The interference of these diagrams can give rise to excess $(B-L)$ number if the loop integral is imaginary . The magnitude of the asymmetry generated by the left-handed triplet $\Delta_{L}$ decay is given by,

$$
\epsilon_{\Delta} \approx \frac{1}{8 \pi\left|f_{L i j}\right|^{2}} \operatorname{Im}\left[h^{*} f_{L i j}^{*} f_{i k} f_{j k}\right] F\left(\frac{h^{*}}{f_{R k k}}\right),
$$

where, $h=\gamma_{12}+\gamma_{21}$ and $F(q)=\ln \left(1+1 / q^{2}\right)$. In general, the quantity $\left[h^{*} f_{L i j}^{*} f_{i k} f_{j k}\right]$ can contain a, rephasing invariant, $C P$ violating phase and so can be complex and the loop integral is imaginary.

For the right handed neutrino $\nu_{R}$ decays, there are two one loop diagrams, which interfere with the tree level diagram. The interference of the tree level diagram with the one with higgs triplet in the loop gives a contribution equal in magnitude to that of the triplet higgs decays. The magnitude of the lepton asymmetry generated from the interference of the tree level diagram with the other loop diagram is given by,

$$
\epsilon_{\nu} \approx \frac{1}{4 \pi\left|f_{i k}\right|^{2}} \operatorname{Im}\left[f_{i k} f_{i l} f_{j k}^{*} f_{j l}^{*}\right] \frac{f_{R i i}}{f_{R k k}} .
$$

The total asymmetry generated is the sum of all these contributions and we denote it as $\epsilon$. 
For the generation of the $(B-L)$ asymmetry we require another ingredient, namely, the $(B-L)$ number generating interactions should not take place in equilibrium [13]. This constraint is extremely stringent [14. Consider the scenario when the right handed electron neutrino is the lightest of the right handed neutrinos and also lighter than the triplet higgs. In that case the $(B-L)$ asymmetry will be generated by the $\nu_{e R}$ if it satisfies the out-of-equilibrium condition. On the other hand if $\nu_{e R}$ does not satisfy the out-of-equilibrium condition, then the $(B-L)$ number violating $\nu_{e R}$ decays will erase any existing $(B-L)$ asymmetry. In that case the sphaleron processes will erase any existing $(B+L)$ asymmetry leaving a baryon symmetric universe causing annihilation catastrophfe. Of course, then one has to think of generating baryon asymmetry during the electroweak phase transition. But with the present limit on the higgs mass such possibilities have also been ruled out.

The out-of-equilibrium condition for the total decay rate of the $\nu_{e R}$ can be written as,

$$
\Gamma_{\nu_{e}} \sim \frac{f_{11}^{2}}{16 \pi} M_{\nu_{e R}} \leq 1.7 \sqrt{g} \frac{T^{2}}{M_{P l}} \quad \text { at } \quad T=M_{\nu_{e}}
$$

For, $f_{11} \sim 10^{-5}$, we get,

$$
M_{\nu_{e R}} \geq 10^{6} \mathrm{GeV}
$$

There are other scattering processes whose rates should also be less than the expansion rate of the universe, such as, $\nu_{e R} e_{R} \rightarrow \bar{u}_{R} d_{R}$. Combining all these bounds, we can obtain a bound on the $S U(2)_{R}$ symmetry breaking scale [11],

$$
M_{R} \geq 10^{7} \mathrm{GeV}
$$

It will then mean that if we see any signature of the right handed gauge bosons at energies much below this scale, then we have to think of new mechanisms for the generation of the baryon asymmetry. 
In this article we argue that for the choice of higgs scalars we have in this model, it is possible to make the left-right symmetry breaking phase transition to be first order and hence generate $(B-L)$ asymmetry during this symmetry breaking even if the symmetry breaking scale is around $\mathrm{TeV}$ and does not satisfy the out-of-equilibrium condition. For this purpose we show that after intregating out the quadratic part, the finite temperature effective potential for this model at the one loop level contains linear terms in $T$, which after minimization can make this phase transition a first order [15].

For this purpose we will introduce thermal field theory and the concept of thermal effective action in the imaginary time formalism of Matsubara. We compute the partition function $Z$ and change the time coordinate $t$ over to a variable defined as $\tau=i t$ with the limits of integration varying between 0 to $\beta$ and perform the functional integration over the fields $($ e.g $\phi(\tau, x))$ with a periodic (antiperiodic) boundary conditions in $\tau$ for bosons (fermions).

For a Lagrangian that is quadratic in the field variables, one can compute the partition function $\mathrm{Z}$ exactly by expanding the field variables e.g $\phi(t, x)$ as

$$
\phi(\beta, x)=\frac{1}{\beta} \sum_{n} \int \frac{d^{3} p}{(2 \pi)^{3}} e^{-i\left(\omega_{n} \tau-p . x\right)} \phi\left(\tilde{\omega_{n}}, p\right)
$$

and performing the Gaussian integration over the field variables. Here $\omega_{n}=$ $\frac{2 \pi n}{\beta}(n=-\infty$ to $\infty)$ are the (Matsubara) frequencies for bosons and have been defined to agree with the periodic boundary conditions of the field variables but otherwise one has to perform expansion and compute the quantities to a certain order in perturbation theory. Starting from the partition function $Z$, we define the effective action in the presence of a constant background field $\bar{\Gamma}\left(\phi_{c}\right)$ in terms of the Legendre transform [17 of the generating functional for the connected Green's function $W^{\beta}(J)=\ln Z^{\beta}(J)$ as

$$
\bar{\Gamma}\left(\phi_{c}\right)=W^{\beta}[J]-\int d \bar{x} \phi_{c}(\bar{x}) J(x)
$$


where $\phi_{c}(\bar{x})$ is the classical field defined as $\phi_{c}(\bar{x})=\frac{\delta W^{\beta}[J]}{\delta J[\bar{x}]}$ and the source is given by $J(\bar{x})=-\frac{\delta \Gamma\left[\phi_{c}\right]}{\delta \phi_{c}[\bar{x}]}$, where the vector $\bar{x}=(-i \tau, \vec{x})$.

The quantity $\bar{\Gamma}\left(\phi_{c}\right)$, evaluated semiclassically about some field configuration $\phi_{c}(\bar{x})$, gives the free energy of the system in that configuration. The effective potential $V_{\text {eff }}(\phi)$, which is the first term in a derivative expansion of $\bar{\Gamma}(\phi)$, is the free energy density in a background constant field configuration. We shall study the effective potential for determining the order of the phase transition for the model under consideration. In the case of a first order phase transition, usually signalled by the presence of a $\phi^{3}$ term in the effective potential, a system can be trapped temporarily in a metastable state leading to non-equilibrium phenomena. The rate of decay for such a system is determined from the imaginary part of its free energy [18] or effective potential. We have investigated this in the one loop approximation at high temperature. With the choice of Higgs fields under consideration, the Lagrangian is given by

$$
\mathcal{L}=\mathcal{L}_{\text {gaugefield }}+\mathcal{L}_{\text {Higgs }}+\mathcal{L}_{\text {fermions }}
$$

The gauge field part of the lagrangian contains the kinetic energy terms for the gauge bosons corresponding to the gauge groups $S U(2)_{L} \times S U(2)_{R} \times$ $U(1)_{B-L}$. The gauge coupling constants for the gauge groups $S U(2)_{L}$ and $S U(2)_{R}$ are same and we denote it by $g$, while that of the $U(1)_{B-L}$ is denoted by $g^{\prime}$. The higgs part of the lagrangian contains the kinetic energy terms for the fields $\Delta \mathrm{s}$ and the field $\phi$, and the scalar interaction terms given by equation 2. The fermionic part of the lagrangian contains the kinetic energy terms for the fermions and the Yukawa couplings given by equation 1. The symmetry breaking scales that we consider are $\langle\phi\rangle:\left(k^{2}+k^{\prime 2}\right)^{\frac{1}{2}} \simeq$ $250 \mathrm{GeV} ; \quad\left\langle\Delta_{R}\right\rangle: v_{R} \simeq 1-10 \mathrm{TeV}$ and $\left\langle\Delta_{L}\right\rangle: v_{L} \simeq 10^{-1}-10^{-5} \mathrm{GeV}$.

We have computed the effective potential at the one loop level, in the Feynman gauge by integrating out the quadratic field variables present in 
the Lagrangian. Upon doing so we have arrived at integrals of the form

$$
I_{ \pm}(k)=\int_{0}^{\infty} d k k^{2} \ln \left[1 \mp e^{\beta \sqrt{k^{2}+M^{2}}}\right]
$$

where $I_{+}$is used for bosons and $I_{-}$is used for fermions. From here, following Dolan and Jackiw [16], we have evaluated these integrals in the high temperature limit. In this limit the effective potential can be written as

$$
V=V_{\text {tree }}+V_{T=0}+V_{T}
$$

Where

$$
V_{T} \simeq A \frac{\pi^{2} T^{4}}{90}+B v_{R}^{2} T^{2}-C T+D \ln \left(v_{R} T\right)^{2}+E
$$

and $\mathrm{A}, \mathrm{B}, \mathrm{C}, \mathrm{D}$ and $\mathrm{E}$ are the functions of the parameters present in the theory. The first term is from the familiar ultra-relativistic limit of a gas of bosons or fermions, the other terms are the corrections to it, coming from the interactions present in the theory. The term $\mathrm{E}$ is the one containing zero temperature effects. The term linear in $\mathrm{T}$ is the one we are interested in, because that is the one responsible for first order phase transition, and it is given by

$$
\begin{gathered}
C=\frac{2 \pi}{3}\left[\left(m_{Z_{L}}^{3}+m_{Z_{R}}^{3}+2 m_{W_{L}}^{3}+2 m_{W_{R}}^{3}\right)+\frac{1}{16 \pi}\left(3 M^{3}+2 M_{R 1}^{3}+M_{R 2}^{3}\right)\right. \\
\left.+\frac{1}{8 \pi}\left(\Sigma_{a=1}^{3} M_{\phi a}^{3}\right)\right]
\end{gathered}
$$

Here

$$
\begin{aligned}
M_{R 1}{ }^{2} & =\mu_{\Delta_{L}}{ }^{2}+2 \overline{\lambda_{R}} v_{R}{ }^{2} \\
M_{R 2}{ }^{2} & ={\mu_{\Delta_{L}}}^{2}+4{\overline{\lambda_{R}}}_{R}{ }^{2} \\
M^{2} & ={\mu_{\Delta_{L}}}^{2}+{\overline{\lambda_{R}}}^{2} v_{R}
\end{aligned}
$$

and

$$
M_{\phi_{1}}{ }^{2}=\left[m^{2}+3 \lambda_{\phi}^{-}\left(k^{2}+k^{\prime 2}\right)+2 \lambda_{L R}^{-} v_{R}^{2}+2\left(\lambda_{L R}^{\overline{1}} v_{R} v_{L}\right)\right]
$$




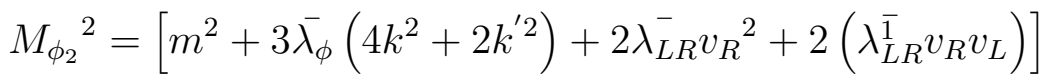

$$
\begin{aligned}
& {M_{\phi_{3}}}^{2}=\left[m^{2}+3{\overline{\lambda_{\phi}}}^{-}\left(2 k^{2}+4 k^{\prime 2}\right)+2{\overline{\lambda_{L R}}}^{-} v_{R}^{2}+2\left(\lambda_{L R}^{\overline{1}} v_{R} v_{L}\right)\right]
\end{aligned}
$$

and

$$
\overline{\lambda_{\phi}}=-\lambda_{\phi}
$$

where, we have defined $\lambda_{L}=\left(\rho_{1}+\rho_{2}\right) ; \lambda_{\gamma}=\gamma_{12}+\gamma_{21}$ and $\lambda_{L R}=\left(\alpha_{12}+\right.$ $\alpha_{21}+\beta_{12}+\beta_{21}$ ). For a range of choice of parameters ( $\lambda$ 's are usually taken to be less or equal to one) the Left-Right symmetry breaking phase transition can be made to be of first order. It is worth pointing out here that since the symmetry breaking scale for the right handed particles is greater than all the other mass scales present in the theory, this one loop result has an imaginary piece in it, that signifies decay of the system from symmetric to the asymmertic vacuum through tunneling. This decay rate is proportional to the imaginary part of the effective potential. One can improve the result of this analysis in the infrared region by summing up the daisy diagrams but according to the standard folklore that only introduces a factor of $\frac{2}{3}$ in front of the cubic term, and hence makes the transition weakly first order. Some of these studies (both numerical and analytical) are currently under consideration.

In the $(B-L)$ broken phase, most of the fermionic fields are massless. The source of $C P$ violation in this phase is through the Majorana mass terms of the right handed neutrinos [7, 8, 9]. As it has been mentioned previously, in this sector there is enough $C P$ violation and the amount of lepton number asymmetry generated is given by

$$
\langle\mathcal{O}\rangle=\frac{2 \epsilon_{\Delta}+\epsilon_{\nu}}{g_{*}}
$$

where, $g_{*}$ is the effective numbers of massless degrees of freedom and after the left-right symmetry breaking it is $g_{*} \sim 400$; To obtain the amount of 
lepton number asymmetry inside this domain wall one has to solve the Boltzmann equation. However, in the limit of large time, the asymptotic order of magnitude for the solution of the Boltzmann equation can be estimated following earlier references [7] to be as given by the above expression. On the other hand, in the $(B-L)$ conserving phase there is no $\mathrm{CP}$ violation since all fermion masses (including the Majorana masses of the right handed neutrinos) vanish. In this phase, one can then rephase the fermion fields to absorb all the phases. We denote the thickness of the domain walls which separates these two phases by $l$, which is related to the inverse of the time derivative of the scalar operator for the field $\Delta_{R}$. The detail calculation of the sphaleron transition probability between the two phases will depend on the wall thickness. An order of magnitude estimate for the lepton number asymmetry thus generated is given by [5],

$$
\frac{n_{L}}{s} \approx \frac{1}{g_{*}}\left(\frac{g^{2}}{4 \pi}\right)^{4} l\langle\mathcal{O}\rangle .
$$

The observed baryon asymmetry is then generated from this $(B-L)$ asymmetry during the electroweak phase transition, during the epoch when the $(B+L)$ number violating interactions are in equilibrium in the universe, and is given by [19,

$$
n_{B}=-\left(\frac{8 N_{g}+4 N_{H}}{22 N_{g}+13 N_{H}}\right) n_{(B-L)} \approx \frac{1}{3} n_{L} .
$$

For a wide range of choice of parameters this can give the required amount of baryon asymmetry, since the parameters in the left-right symmetric model are not constrained by phenomenology. Consider the expression for the $\epsilon \mathrm{s}$, where only the Yukawa couplings of the right handed neutrinos enter. But both the Majarana as well as the Dirac masses of these particles are free parameters and one can choose a large combination of these parameters, which can reproduce the amount of baryon asymmetry required by the standard big-bang model. 
To summarize, we have studied the effective potential of the left-right symmetric model at finite temperature and have shown that the left-right symmetry breaking can take place through first order phase transition at around $\mathrm{TeV}$ scale. This can then generate enough $(B-L)$ asymmetry, which can then generate enough baryon asymmetry during the electroweak phase transition.

Acknowledgement One of us (US) would like to acknowledge a fellowship from the Alexander von Humboldt Foundation and hospitality from the Institut für Physik, Univ Dortmund during his research stay, where part of this work was done.

\section{Figure Caption}

Figure 1 Diagrams contributing to the generation of lepton asymmetry. 


\section{References}

[1] E.W. Kolb and M.S. Turner, The Early Universe (AddisonWesley, Reading, MA, 1989).

[2] P. Langacker, Phys. Rep. 72 (1981) 185; A. Masiero in "Grand Unification with and without Supersymmetry and Cosmological Implications", International School for Advanced Studies Lecture Series No. 2, published by World Scientific, 1984, p-1; and A. Zee (ed.) "Unity of Forces in the Universe", Vol. (1,2), published by World Scientific, 1982.

[3] G. 't Hooft, Phys. Rev. Lett. 37 (1976) 8; V.A. Kuzmin, V.A Rubakov and M.E. Shaposhnikov, Phys. Lett. B 155 (1985) 36.

[4] M.E. Shaposnikov, Nucl. Phys. B 287 (1987) 757; B 299 (1988) 797; N. Turok and J. Zadrozny, Phys. Rev. Lett. 65 (1990) 2331; A. Kazarian, S. Kuzmin and M.E. Shaposnikov, Phys. lett. B 276 (1992) 131.

[5] L. McLerran, M.E. Shaposnikov, N. Turok and M. Voloshin, Phys. lett. B 256 (1991) 451;

[6] A.I. Bochkarev, S.V. Kuzmin and M.E. Shaposnikov, Phys. Lett. 244 B (1990) 275; M. Dine, P. Huet and R. Singleton Jr., Nucl. Phys. B 375 (1992) 625.

[7] M. Fukugita and T. Yanagida, Phys. Lett. B 174 (1986) 45; P. Langacker, R.D. Peccei and T. Yanagida, Mod. Phys. Lett. A 1 (1986) 541; M.A. Luty, Phys. Rev. D 45 (1992) 445; H. Murayama, H. Suzuki, T. Yanagida and J. Yokoyama, Phys. Rev. Lett. 70 (1993) 1912. 
[8] P.J. O'Donnell and U. Sarkar, Phys. Rev. D 49 (1994) 2118.

[9] A. Acker, H. Kikuchi, E. Ma and U. Sarkar, Phys. Rev. D 48 (1993) 5006.

[10] J.C. Pati and A. Salam, Phys. Rev. D10 (1974) 275; R.N. Mohapatra and J.C. Pati, ibid. D11 (1975) 566; R.N. Mohapatra and G. Senjanović, Phys. Rev. Lett. 44 (1980) 912; R.E. Marshak and R.N. Mohapatra, ibid. 44 (1980) 1316.

[11] R.N. Mohapatra and X. Zhang, Phys. Rev. D 46 (1992) 5331.

[12] The present lower bound on the right handed gauge boson mass is only around $500 \mathrm{GeV}$. See, e.g., J. Freeman, in 'Particle Phenomenology in the 90's', eds. A. Datta, P. Ghose and A. Raychaudhuri (World Scientific, 1992); G. Bhattacharyya, A. Datta, S.N. Ganguli and A. Raychaudhuri, Phys. Rev. Lett. 64 (1990) 2870; Mod. Phys. Lett. (Brief Review) A6, (1991) 2557; G. Altarelli et al., Phys. Lett. B245 (1990) 669; Nucl. Phys. B342 (1990) 15; Mod. Phys. Lett. A5 (1990) 495; G. Bhattacharyya, A. Datta, A. Raychaudhuri and U. Sarkar, Phys. Rev. D 47 (1993) R3693.

[13] S. Weinberg, Phys. Rev. Lett. 42 (1979) 850; D. Toussaint, S.B. Treiman, F. Wilczek and A. Zee, Phys. Rev. D 19 (1979) 1036; J.N. Fry, K.A. Olive and M.S. Turner, Phys. Rev. Lett. 45 (1980) 2074; Phys. Rev. D 22 (1980) 2953; ibid. D 22 (1980) 2977.

[14] M. Fukugita and T. Yanagida, Phys. Rev. B 42 (1990) 1285; B.A. Campbell, S.Davidson, J. Ellis and K.A. Olive, Phys. Lett. B 256 (1991) 457; A.E. Nelson and S.M. Barr, Phys. Lett. B 246 (1990) 141. 
[15] J. Choi and R.R. Volkas, Phys. Rev. D 48, (1993) 1258.

[16] L. Dolan and R. Jackiw, Phys. Rev. D 9 (1974) 3320; S. Weinberg, ibid. D 9 (1974) 3357.

[17] C.V. Bernard, Phys. Rev. D 9 (1974) 3312.

[18] I. Affleck, Phys. Rev. Lett. 46 (1981) 388; J.S. Langer, Ann. Phys. (N.Y.) 41 (1867) 108.

[19] J.A. Harvey and M.S. Turner, Phys. Rev. D 42 (1990) 3344. 
This figure "fig1-1.png" is available in "png" format from: http://arxiv.org/ps/hep-ph/9408271v1 